\title{
Pitfalls in Imaging of Female Pelvic Masses
}

\author{
Mariana Horta ${ }^{1,2}$ Teresa Margarida Cunha ${ }^{1}$
}

(C) Springer Science+Business Media, LLC 2017

\begin{abstract}
Purpose of Review The purpose of this review is to highlight potential magnetic resonance (MR) imaging pitfalls that may mask and simulate ovarian cancer.

Recent Findings MR imaging is the standard method for evaluating female pelvic masses of indeterminate origin, especially sonographically indeterminate adnexal masses. To define the correct origin and nature of a pelvic mass has an enormous clinical impact, namely in females of child-bearing age. This is particularly true in adnexal lesions. Ovarian cancer usually requires a cytoreductive surgery in a specialized oncological centre. In contrast, a benign ovarian lesion may be treated by simple resection in a general hospital.

To help preventing diagnostic errors and in order to guide appropriate therapeutic management, radiologists should be aware of potential MR pitfalls that may mask and simulate ovarian cancer.

Summary The first section of this article will describe the MR imaging protocol that authors perform in their oncological centre, highlighting how imaging techniques can be optimized in order to reduce pitfalls in the characterization of an indeterminate pelvic mass.
\end{abstract}

This article is part of the Topical Collection on Urogenital Imaging.

Mariana Horta

mariana.sf.horta@gmail.com

Teresa Margarida Cunha

tmargarida@gmail.com

1 Department of Radiology, Instituto Português de Oncologia de Lisboa Francisco Gentil, R. Prof. Lima Basto, 1099-023 Lisbon, Portugal

2 Instituto de Anatomia, Faculdade de Medicina da Universidade de Lisboa, Lisbon, Portugal
In the next section, authors will revise the main anatomic and organ-specific signs that may allow the radiologist to determine the ovarian origin of a pelvic mass.

Finally, benign gynaecological masses that may simulate ovarian cancer in a non-emergency setting will be discussed, with emphasis on features that may provide important clues to their diagnosis.

Keywords Ovarian cancer - Benign ovarian tumours . Magnetic resonance imaging - Female pelvic masses . Indeterminate adnexal masses - Pitfalls in gynaecological imaging

\section{Introduction}

Radiological imaging is a key diagnostic tool of modern clinical diagnosis. It is also an important source of diagnostic errors that can have a major impact in patients' treatment, causing patients harm $[1 \bullet \bullet]$.

Patient management errors have been the most cited in claims ascribed to negligence both in Radiology and in Gynaecology [2].

Major discrepancy of radiological interpretation has been reported amongst experienced radiologists when reading abdomino-pelvic computed tomography (CT) exams [3]. The retrospective inter-observer variability was reported in approximately $26 \%$ and intra-observer discrepancy was also estimated in 32\% [3].

Although technical aspects of radiological exams may be a source of diagnostic errors, the majority of radiological mistakes are perceptual and cognitive/interpretive errors $[1 \bullet \bullet, 4]$.

Perceptual errors are responsible for the majority of diagnostic errors (up to 90\%) [5-7]. They are defined as 

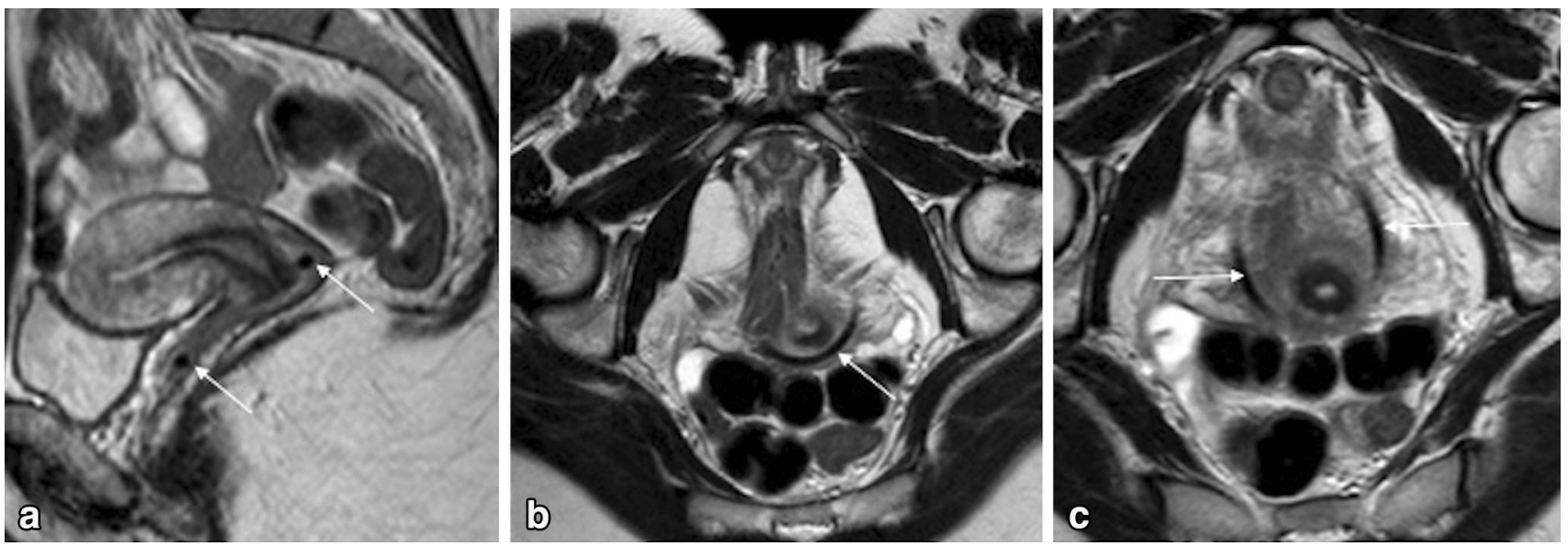

Fig. 1 Pelvic MRI for suspected endometriosis in a 32-year-old woman. a Sagittal T2-weighted image; $\mathbf{b}$ and $\mathbf{c}$ Axial of the cervical canal T2-weighted images. Authors always ask clinical questions before the exam is performed, especially information that disproves

the initial diagnosis. In this case, the patient was using a contraceptive vaginal ring (arrows) that could be misinterpreted as pelvic endometriosis
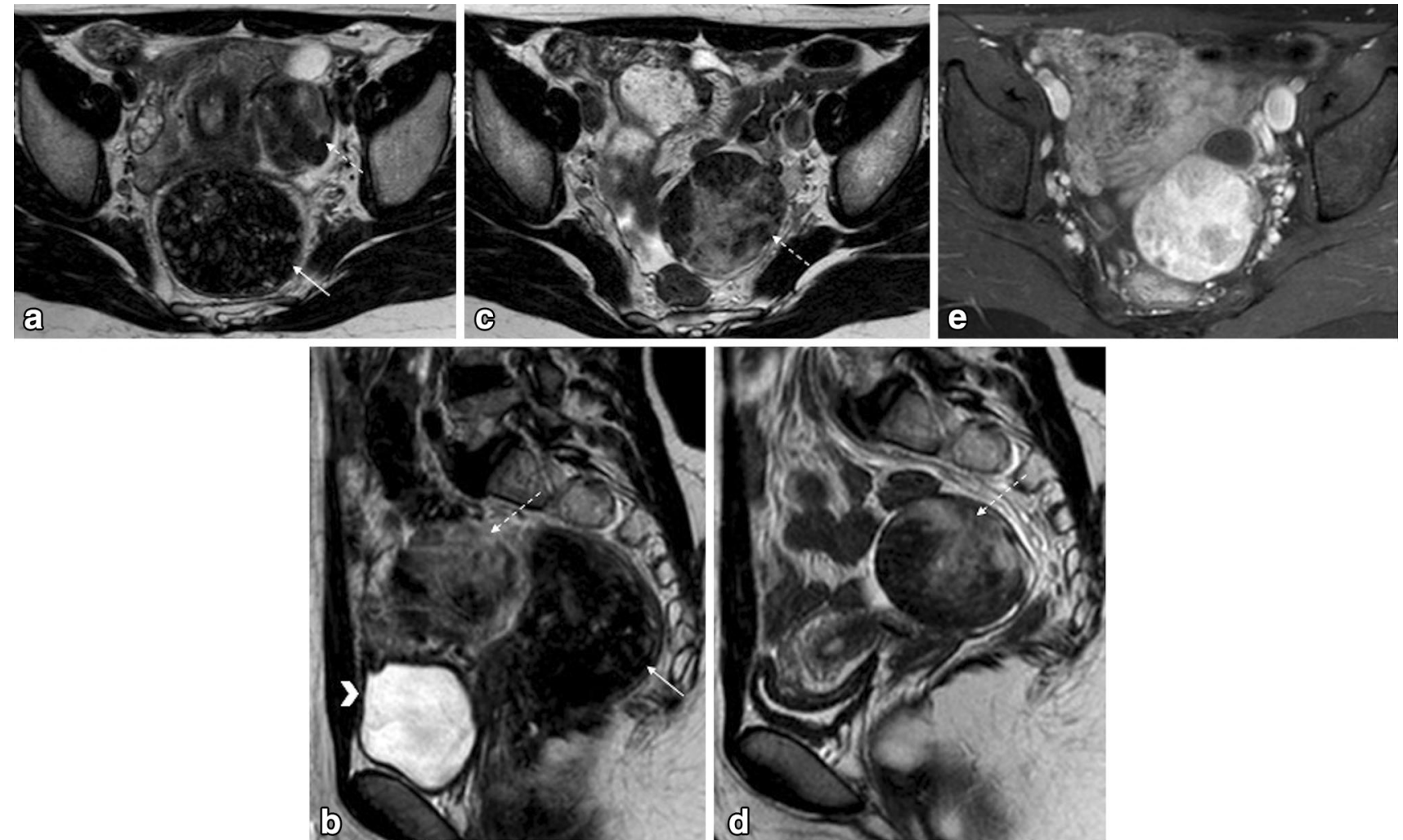

Fig. 2 Pelvic MRI for the characterization of an indeterminate adnexal mass in a 20-year-old woman. a and c Axial T2-weighted images; $\mathbf{b}$ and $\mathbf{d}$ Sagittal T2-weighted images; e Axial fat-suppressed contrast-enhanced T1-weighted image. Authors always encourage patients to evacuate before the exam, usually with the aid of a Microlax ${ }^{\circledR}$. Patients also empty their bladder $1 \mathrm{~h}$ prior to the exam in order to achieve a moderately filled bladder. This case illustrates how rectal stools (arrow) and a filled bladder (arrowhead) impede the correct characterization of gynaecological lesions (a and $\mathbf{b}$ ). After evacuation and voiding, the adnexal mass (dashed-arrows) could be better characterized (c and d). It showed typical features of a sclerosing cell tumour of the ovary (benign tumour). It showed peripheral hypointense nodular areas with a spoke-wheel pattern on T2-weighted images. Dynamic contrast-enhanced study typically revealed an avid and early peripheral uptake that progresses to central areas on delayed images. The patient had a fertility-sparing surgery 


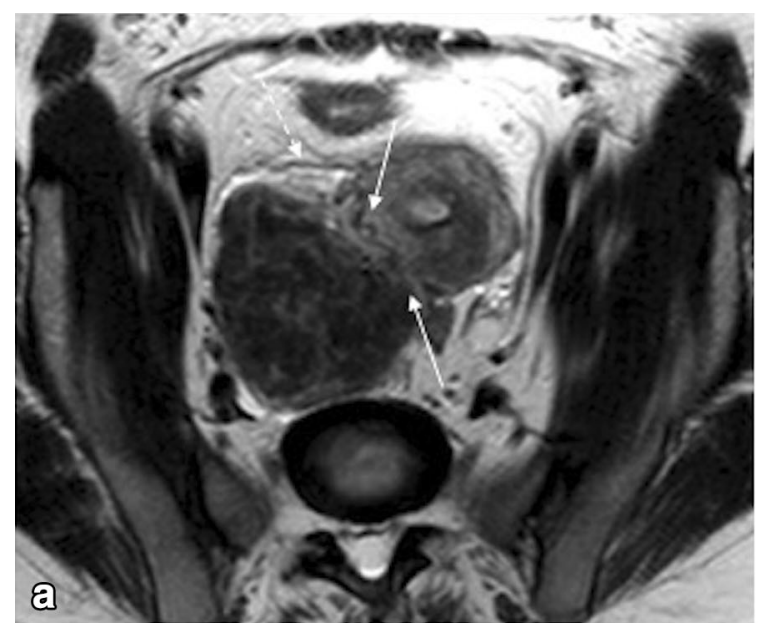

Fig. 3 "Bridging vessel" sign in a 32-year-old woman with a subserosal leiomyoma. a and b Axial of the uterine corpus T2weighted images. In this case the right ovary (dashed-arrow) was compressed by the mass, making it difficult to diagnose the origin of

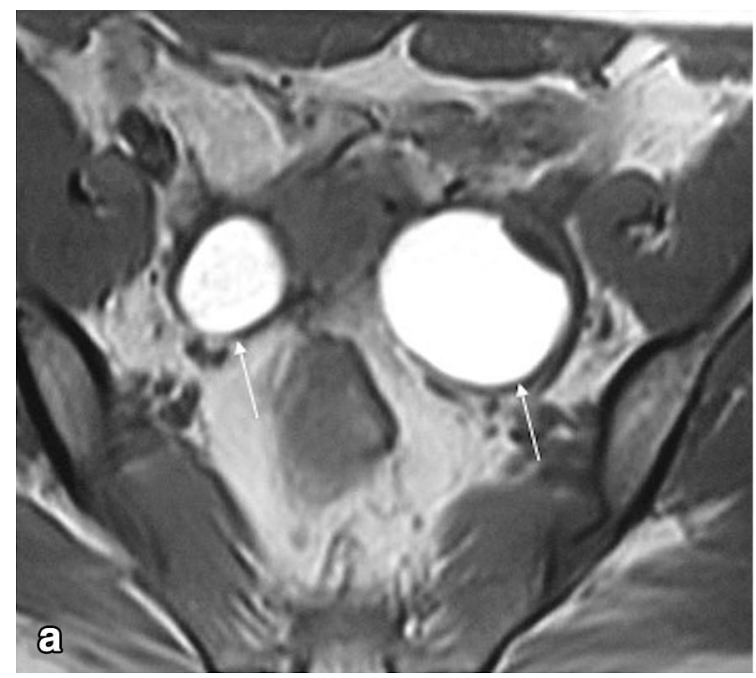

Fig. 4 Bilateral endometriomas in a 44-year-old woman. a Axial T1weighted image; b Axial fat-suppressed T1-weighted image. Hyperintense T1-weighted lesions are seen in both ovaries. In this case a

those in which the radiological abnormality was not seen in the initial interpretation.

Cognitive or interpretative errors are caused by misinterpretation due to faulty reasoning, misleading clinical information, lack of knowledge or propagation of an error that a previous radiologist had made $[1 \bullet \bullet, 8]$.

The misinterpretation of adnexal masses in women who undergo adnexal surgery is common [9].

There is a tremendous clinical impact when an indeterminate adnexal mass is diagnosed as malignant or benign. Malignant ovarian tumours should be referred to a specialized oncological centre to assess if optimal cytoreductive surgery is possible $[10 \bullet \cdot]$. On the other hand,

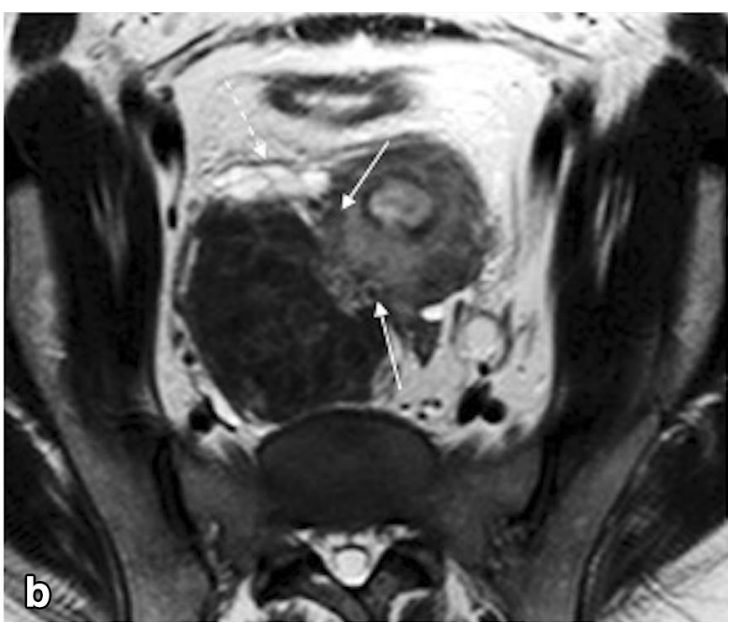

the pelvic mass. In order to determine if the pelvic mass is ovarian or uterine in origin, the authors perform a plane through the point of maximum contact of the mass and the uterus to look for the "bridging vessel" sign (arrows)

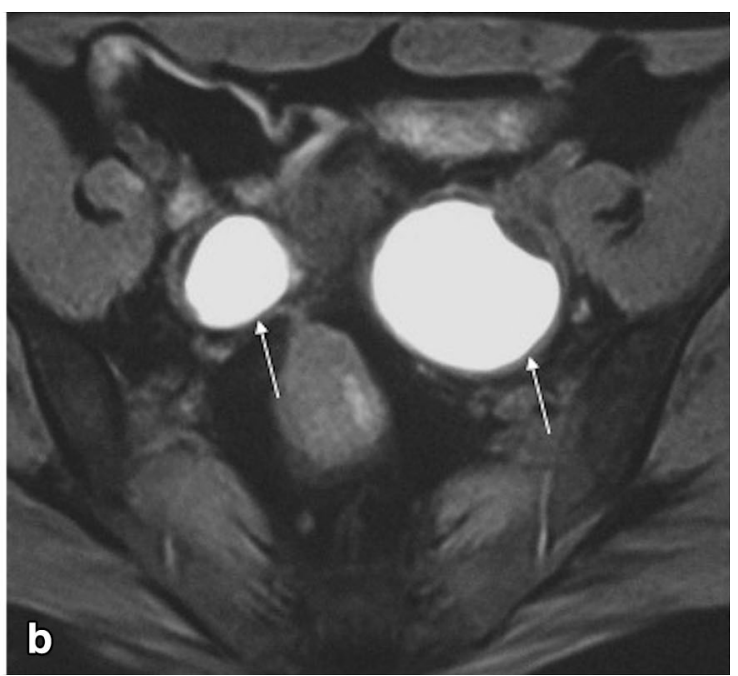

fat-suppressed T1-weighted sequence is mandatory to determine whether it has blood or fat content. No fat suppression is seen, therefore both lesions have blood content

benign tumours can be treated in general hospitals usually by simple resection.

In general hospital and private daily-practices, pelvic gynaecological CT and magnetic resonance (MR) imaging are usually read by general abdominal radiologists with different levels of expertise. Many general radiologists are not familiarized with specific types of adnexal and pelvic tumours, which are frequent in a gynaecological oncological setting [9].

A second-opinion review is being increasingly done by radiologists in order to decrease the rate of error and misinterpretation. One study has addressed the impact of a secondopinion review of gynaecological-oncological MR imaging 

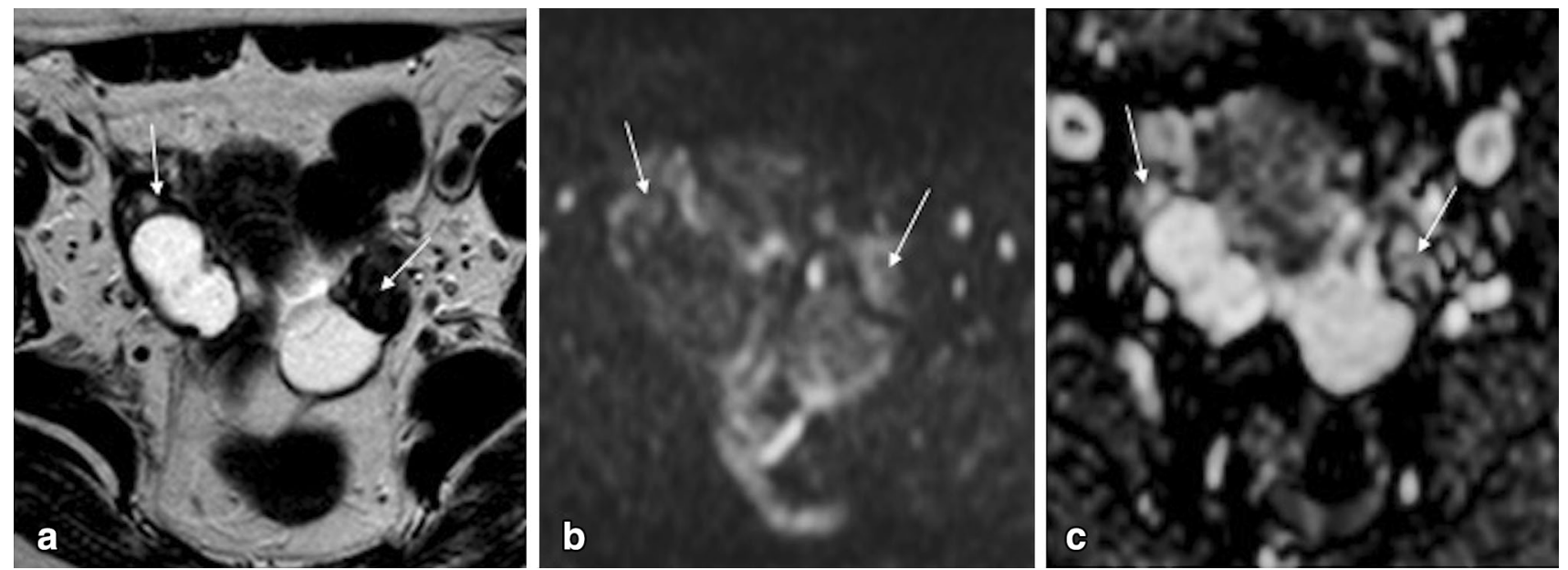

Fig. 5 Bilateral cystadenofibromas in a 56-year-old woman. a Axial T2-weighted image; b Axial diffusion-weighted image $(b=1000 \mathrm{~s} /$ $\mathrm{mm}^{2}$ ); c ADC map. Bilateral mixed ovarian masses are seen. The solid component of these masses shows both low signal intensity on T2- weighted and on DWI images (arrows); thus the lesion is very likely to be benign and fibrotic in nature
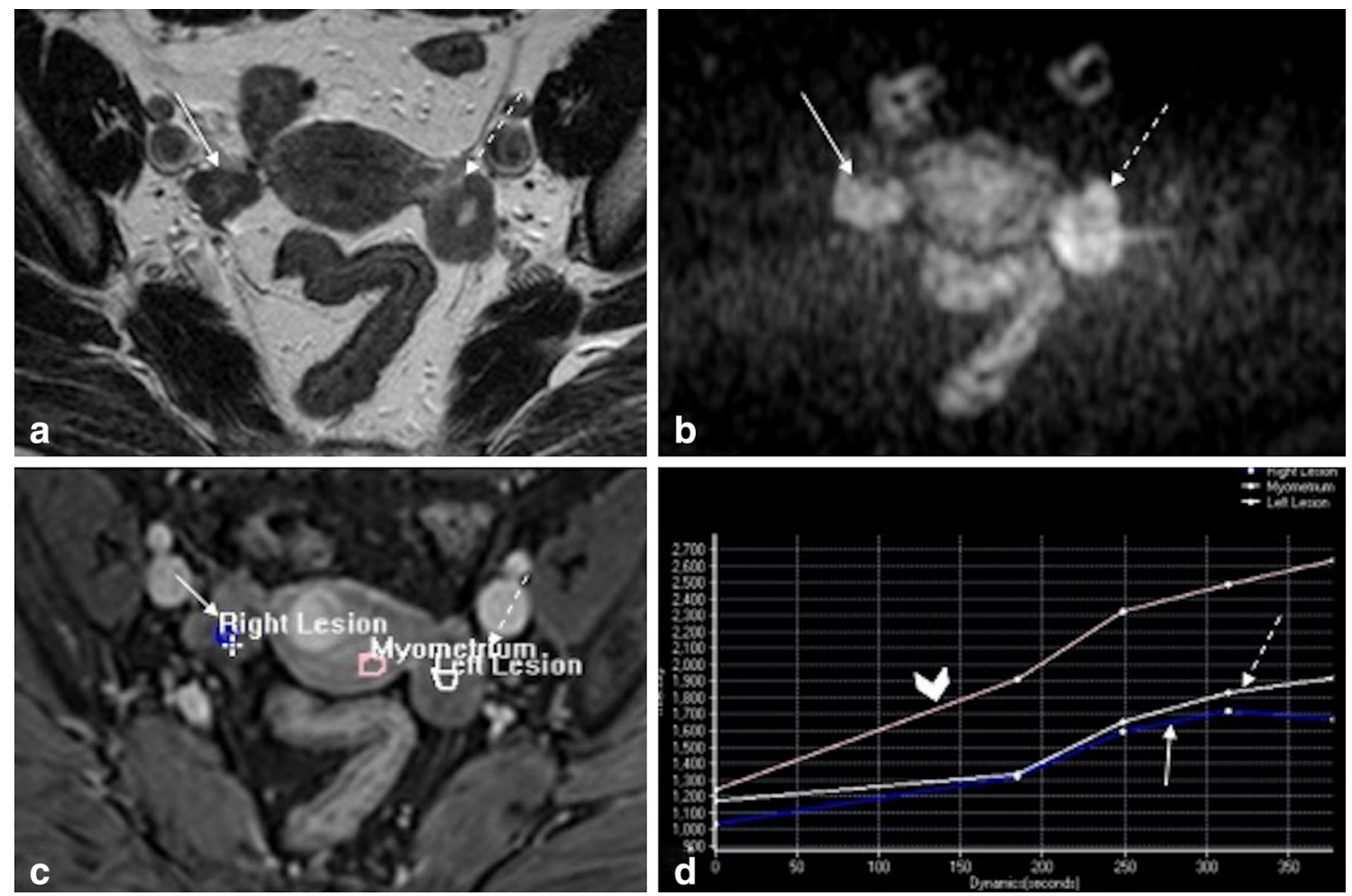

Fig. 6 Bilateral fibromas in a 55-year-old woman. a Axial T2weighted image; b Axial diffusion-weighted image $(b=1000 \mathrm{~s} /$ $\mathrm{mm}^{2}$ ); c Semi-quantitative dynamic contrast-enhanced study; $\mathbf{d}$ Timeintensity curves of the myometrium (arrowhead) and of the right (arrow) and left (dashed-arrow) fibromas. Both ovarian lesions display peripheral low signal intensity on $\mathrm{T} 2$-weighted image and a small central cystic area. However, these tumour also display very

by sub-specialized radiologists of a gynaecological-oncological centre. It has shown that one-fifth of MR imaging had clinically relevant discrepancies and that second-reading was valuable in the management of these patients [11•0].

high signal on DWI image $\left(b=1000 \mathrm{~s} / \mathrm{mm}^{2}\right)$; thus they should be better characterized with the administration of gadolinium. Semiquantitative dynamic study reveals a slow uptake of contrast and a low level of enhancement (type 1 curves) by both lesions, when compared to the myometrium. This curve pattern is very characteristic of benign lesions

The first section of the article will describe the MR imaging protocol that authors perform in their gynaecological oncological centre (Instituto Português de Oncologia de Lisboa Francisco Gentil) for the characterization of a pelvic 

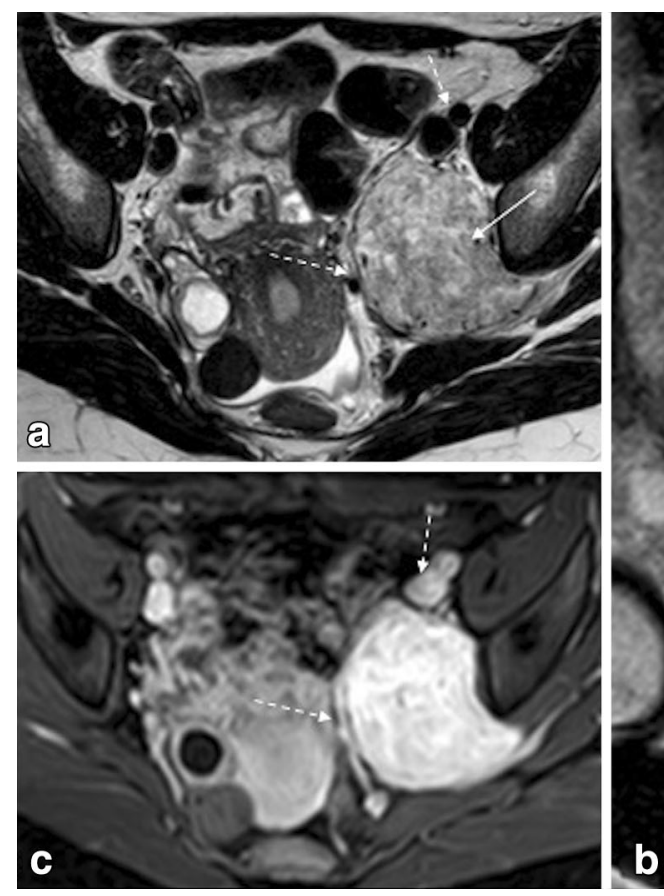

Fig. 7 Left extra-peritoneal solitary fibrous tumour in a 26- year-old woman. a Axial T2-weighted image; b Coronal T2-weighted image; c Axial fat-suppressed contrast-enhanced T1-weighted image. An extra-peritoneal mass (arrow) is seen displacing the vessels anteriorly and laterally (dashed-arrows) and the uterus laterally. There is also effacement of the pelvic wall muscles mass of indeterminate origin. Authors will also address how imaging techniques can be optimized in order to reduce pitfalls in assessing pelvic gynaecological masses.

The next section will cover the potential pelvic anatomical pitfalls. We will revise the main radiological signs that may allow the radiologist to determine the origin of a pelvic mass.

Finally, we will demonstrate how benign gynaecology masses can mimic malignant ovarian tumours in a nonemergency setting.

\section{MR Imaging Technique to Reduce Pitfalls in Imaging Indeterminate Pelvic Masses}

Transvaginal ultrasound complemented with transabdominal approach is the first imaging modality for the assessment of women with a suspected gynaecological mass [12].

According to the European Society of Genitourinary Radiology (ESUR) guidelines, pelvic MR imaging should be performed to further characterize any sonographically indeterminate mass [13••]. Indeterminate adnexal masses are considered those that are complex masses with equivocal malignant features, solid masses, large pelvic masses and masses adjacent to the uterus of unknown origin [13••].

The gynaecological MR imaging protocol of Instituto Português de Oncologia de Lisboa Francisco Gentil follows the ESUR recommendations for the characterization of sonographically indeterminate adnexal masses [13••].

Clinical questions are always asked before the exam (including age, questions regarding clinical symptoms, hormonal status, hormonal medication, recent surgeries and recent deliveries).Cognitive biases such as anchoring and framing are reduced when the radiologist seeks more clinical information from patients, especially disconfirming information that disproves the initial clinical diagnosis [4] (Fig. 1).

The authors find of uttermost importance the use of strategies that limit motion artefacts. We always use antiperistaltic agents for the improvement of image quality (20 mg butyl-scopolamine or $1 \mathrm{mg}$ of glucagon intramuscularly), unless they are contraindicated. We recommend patients to empty their bladder $1 \mathrm{~h}$ prior to examination in order to achieve a moderately filled bladder. Patients are also encouraged to evacuate before the exam, usually with the aid of a Microlax ${ }^{\circledR}$ (Fig. 2). Patients should fast (3-6 h) especially when intravenous injection of contrast is considered. Scanning the patient in prone position may also help to reduce motion artefacts.

Both $3 \mathrm{~T}$ and $1.5 \mathrm{~T}$ magnets are valuable in the assessment of pelvic masses. According to ESUR guidelines, the basic MR imaging examination protocol should have a sagittal T2-weighted imaging of the pelvis and a pair of T1weighted and T2-weighted images in the same plane 

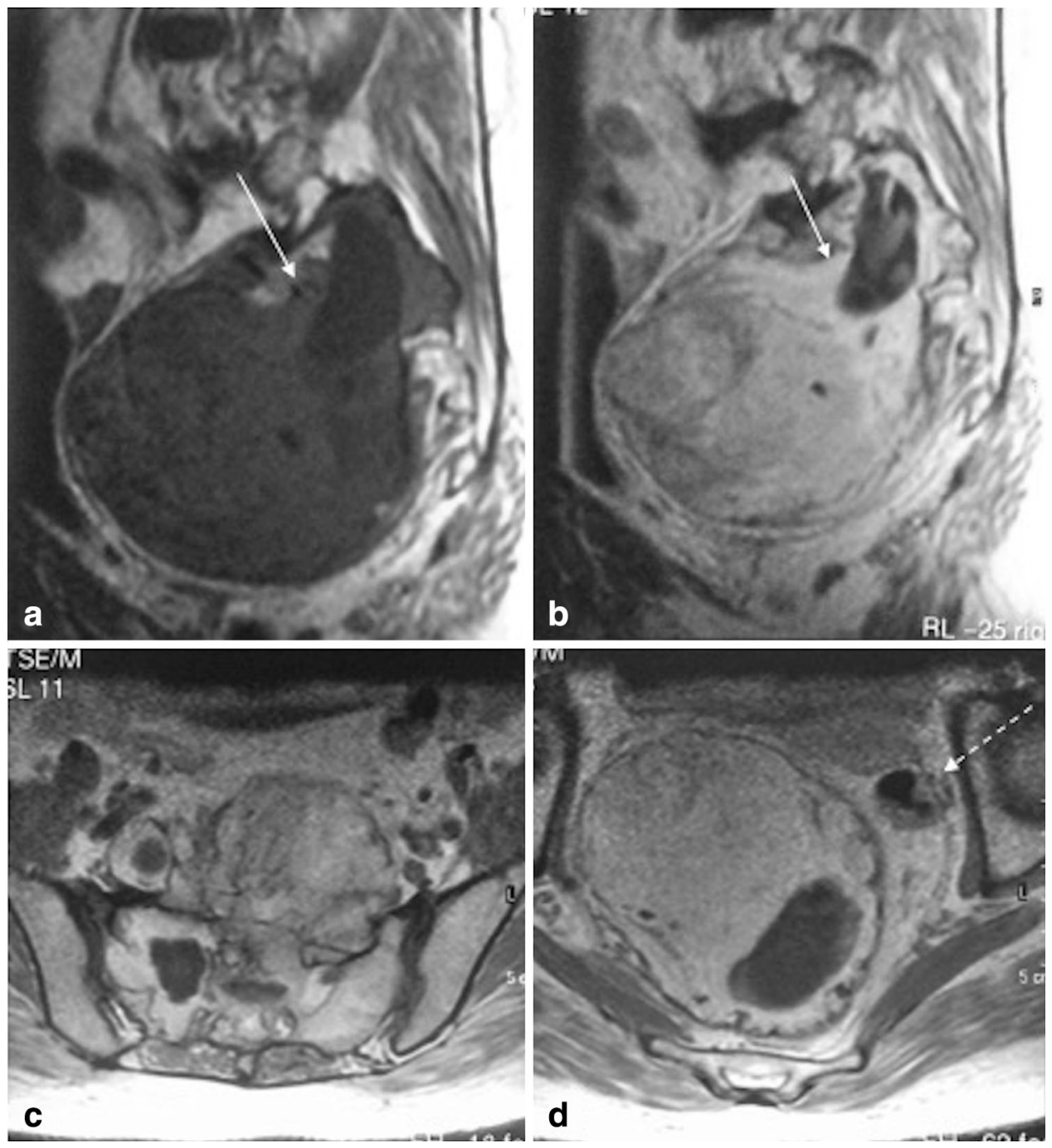

Fig. 8 Extra-peritoneal sacral plexiform schwannoma. a Sagittal T1weighted image; b Sagittal contrast-enhanced T1-weighted image; c and d Axial contrast-enhanced T1-weighted images. An extra-

through the indeterminate mass [13••]. If the origin of the mass is unknown, especially if the mass is adjacent to the uterus, a T2-weighted oblique sequence should be performed along the long axis of the uterus (which is the ovarian axis). Alternatively, a plane through the point of maximum contact of the mass and the uterus should be performed, in order to look for the "bridging vessel" sign that allows to determine the uterine origin of the lesion [13••] (Fig. 3).

When a pelvic mass has high T1 signal, a fat-saturated T1-weighted sequence is mandatory to determine whether it has blood, high-protein or fat content (Fig. 4).

If a pelvic mass has very dark signal on $\mathrm{T} 2$-weighted images, diffusion-weighted images (DWI) should be performed. A mass that displays both low T2 signal and DWI signal $\left(b=800-1000 \mathrm{~s} / \mathrm{mm}^{2}\right)$ is very likely to be benign (ex.: fibroma; cystadenofibroma; pedunculated leiomyoma;

peritoneal highly vascularized mass (arrow) is seen arising from the sacrum. It displaces the rectum anteriorly and laterally (dashedarrow)

Brenner tumour), thus intravenous contrast is not needed [13••, 14] (Fig. 5).

Intermediate T2-weighted solid masses and cystic-solid masses with intermediate to high DWI signal ( $b=800-1000 \mathrm{~s} / \mathrm{mm}^{2}$ ) can be malignant or benign (ex. cellular fibroma, degenerated pedunculated leiomyoma, tubo-ovarian abscess). In this case a T1-weighted sequence with intravenous contrast should be performed [13••] (Fig. 6).

When gadolinium is administered to better characterize a T2-intermediate signal mass or a complex solid-cystic mass, authors perform a semi-quantitative study in order to obtain and compare the time-intensity curve of the solid component of the adnexal lesion with the one of the external myometrium. A rapid uptake of contrast with a rapid washout of the solid components has been strongly associated with malignant lesions (type 3 curve). On the 

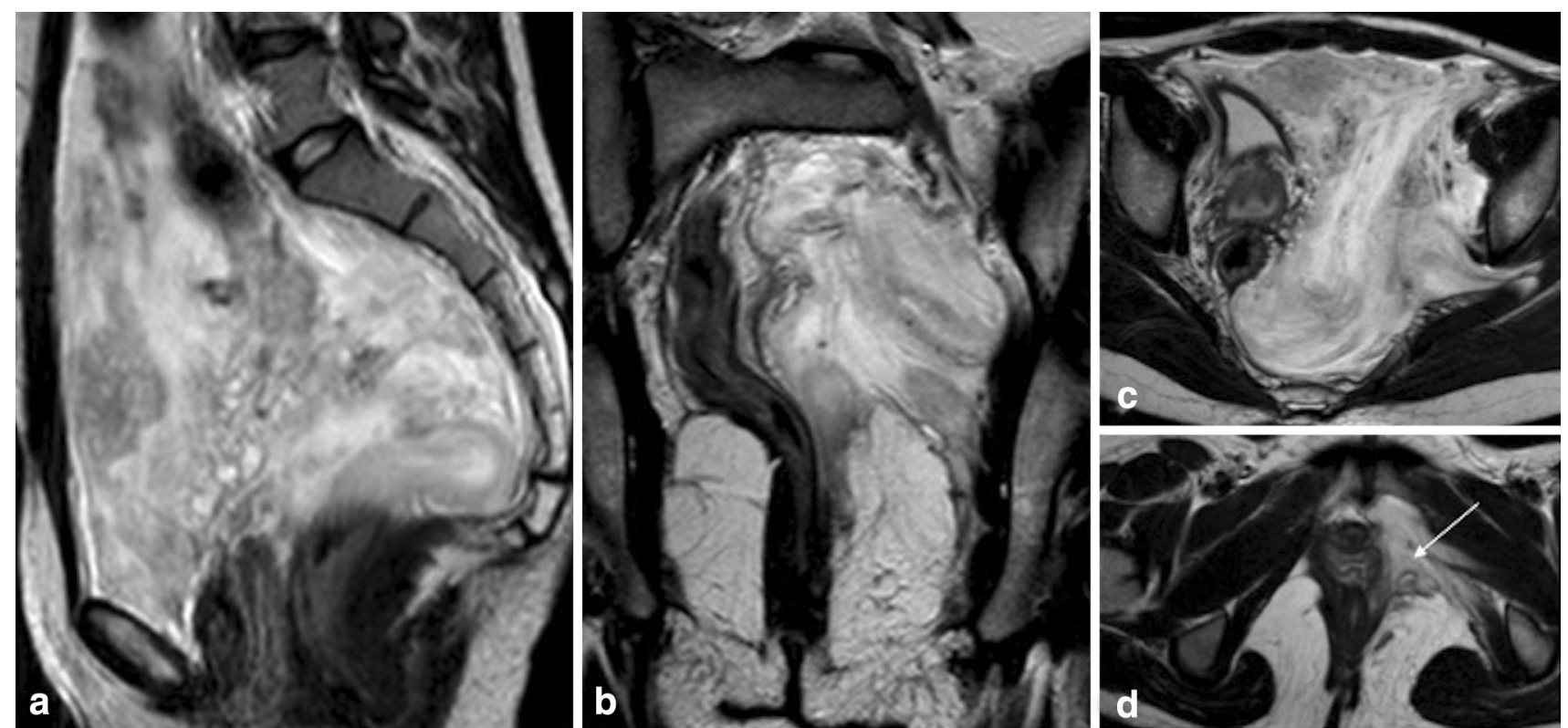

Fig. 9 Vulval aggressive angiomyxoma in a 29 -year-old woman presenting as a transpatial pelvic mass. a Sagittal T2-weighted image; b Coronal T2-weighted image; $\mathbf{c}$ and $\mathbf{d}$ Axial T2-weighted images. A transpatial high T2 signal lesion with a swirling appearance is seen occupying intra and extra-peritoneal spaces (arrows)
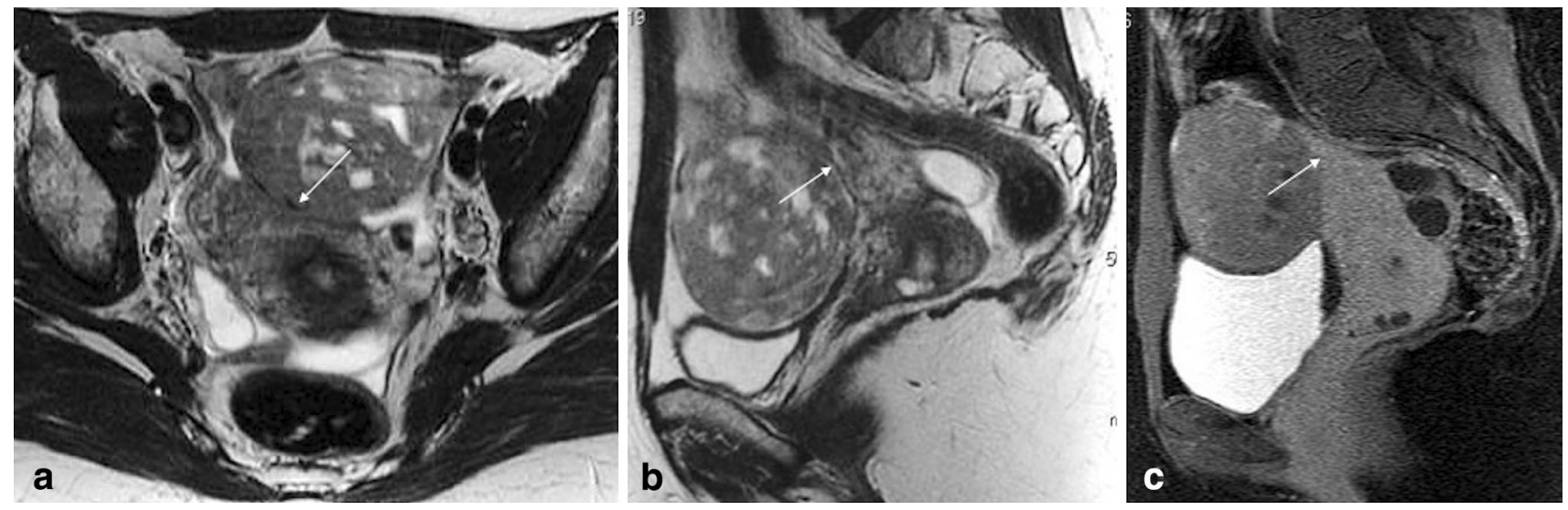

Fig. 10 "Negative embedded organ" sign caused by a small bowel GIST in a 37-year-old woman. a Axial T2-weighted image; b Sagittal T2-weighted image; c Sagittal fat-suppressed contrast-enhanced T1weighted image. Images show an intra-peritoneal pelvic mass that

other hand, a slow uptake of contrast and a low level of enhancement (type 1 curve) has a very high likelihood of benignity [13••, 15-17] (Fig. 6).

\section{Pitfalls in Assessing the Anatomical Origin of a Pelvic Mass}

Large pelvic masses can originate from gynaecological organs and also from the bladder, the upper third of the rectum, the sigmoid colon, the bowel loops and from the extra-peritoneal space. It can be very challenging to define deforms the uterus into a crescent shape ("Negative embedded organ" sign). Thus, this mass could not have an uterine origin. As both the ovaries were depicted (not shown), and walls of the bladder were clearly defined, this mass only could arise from the bowel loops

the correct origin a pelvic mass in order to make a list of differential diagnosis. First, the radiologist should look for the displacement of anatomic landmarks that can help to diagnose the mass as intra- or extra-peritoneal in origin. Then, the radiologist should try to find specific anatomical organ-signs in order to confidently define the mass as ovarian or non-ovarian.

\section{Is it Intra- or Extra-Peritoneal?}

A detailed discussion of the pelvic anatomy is beyond the scope of this article. However, there are some anatomical 

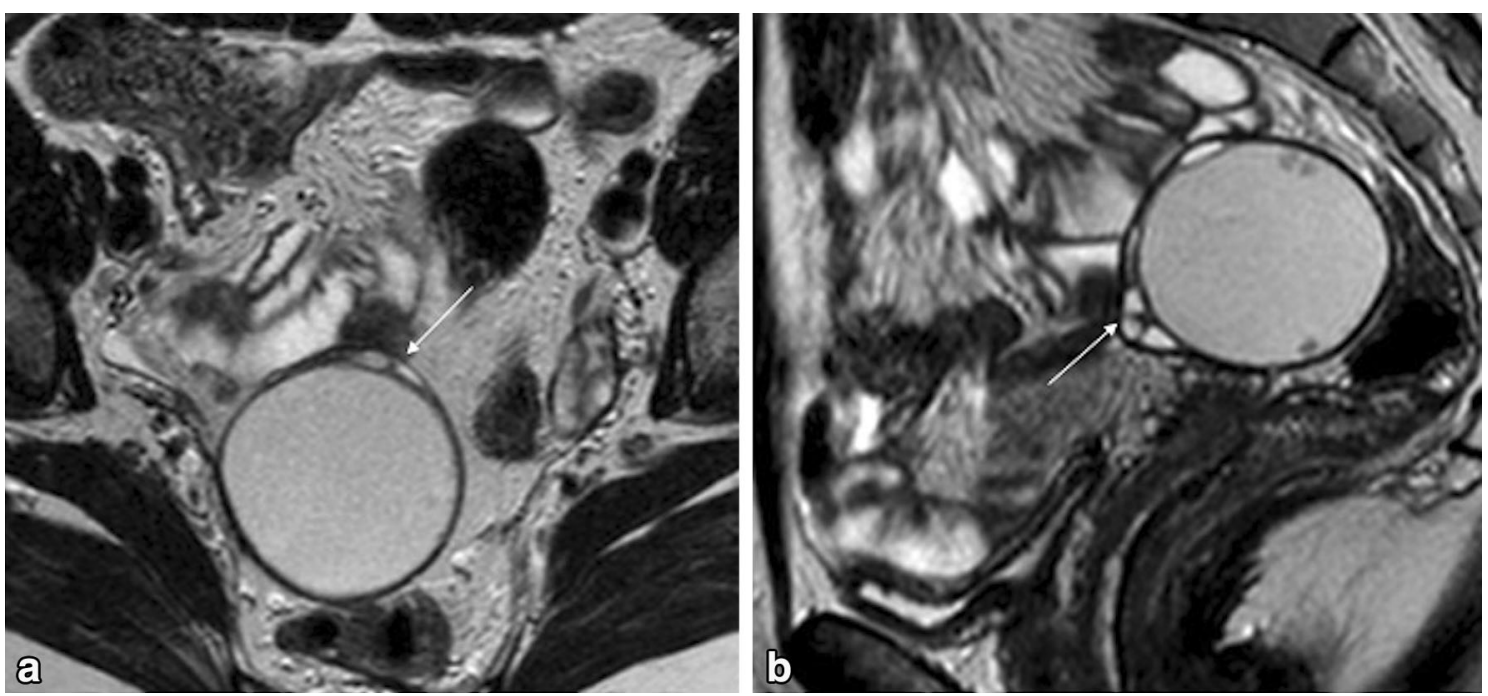

Fig. 11 "Beak" sign a Axial T2-weighted image; b Sagittal T2-weighted image. The "beak" sign occurs when a mass deforms the edge of its organ of origin into a beak shape (arrows)

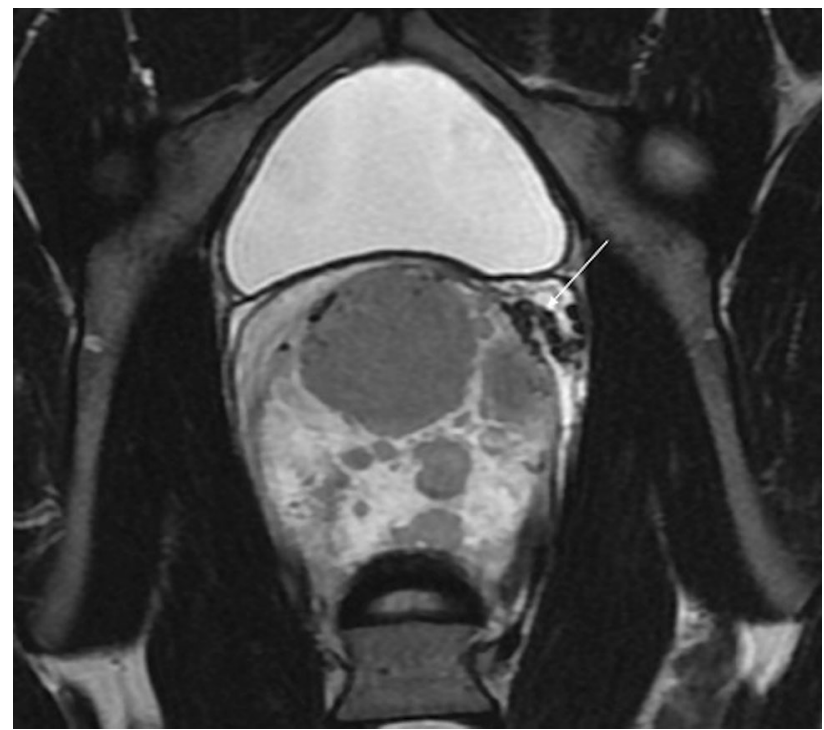

Fig. 12 "Prominent feeding artery" sign caused by a left SertoliLeydig cell ovarian tumour in a 15-year-old girl. Coronal T2weighted image shows an engorged left ovarian artery that allowed the radiologist to diagnose this mass as a left ovarian tumour

concepts that should be kept in mind when we are evaluating a pelvic mass of unknown origin.

The peritoneum is a serous membrane that wraps many of the abdomino-pelvic organs (visceral peritoneum) and covers the walls of the peritoneal cavity (parietal peritoneum). The peritoneal cavity is a potential space formed by the continuous lining of these two layers. In females, the peritoneal cavity opens to the exterior through the fallopian tubes, the uterine and vaginal cavities [18].

An intra-peritoneal organ is anatomically defined as one that is almost completely covered by visceral peritoneum (ex. the uterine corpus, the fallopian tubes, the bladder, the jejunoileum, the sigmoid colon and the upper third of the rectum). Nonetheless, the only organ that is truly intraperitoneal is the ovary, because it is the only one that lies inside the peritoneal cavity making it a non-virtual space. The ovary is not covered by the peritoneum, so the oocyte can reach the fallopian tubes.

An extra-peritoneal structure is one that is not covered by the peritoneum or has only one surface covered by it (ex. the uterine cervix, the vagina, the lower two-thirds of the rectum, the ureter, the iliac vessels and the iliac lymph nodes).

Intra-peritoneal masses typically displace laterally or posteriorly the ureter and the iliac vessels. Conversely, extra-peritoneal masses tend to displace them anteriorly or medially (Fig. 7). An extra-peritoneal mass usually also effaces the pelvic wall muscles (Fig. 7).

If a mass lies in the pre-sacral space it will typically cause anterior and/or lateral displacement and compression of the rectum (Fig. 8).

However, one should note that there are masses that by its aggressive and invasive nature are transpatial (ex.: abscess; hematoma; angiomyxoma); therefore, these patterns of displacement cannot be applied (Fig. 9). In our experience, both intra- and extra-peritoneal masses can cause anterior or lateral displacement of the uterus and bowel, as well as their compression, and thus we do not rely on the pattern of displacement of these organs.

\section{Is it Ovarian or a Non-ovarian Mass?}

There are four radiological signs that may help the radiologist to establish the origin of a pelvic mass. 


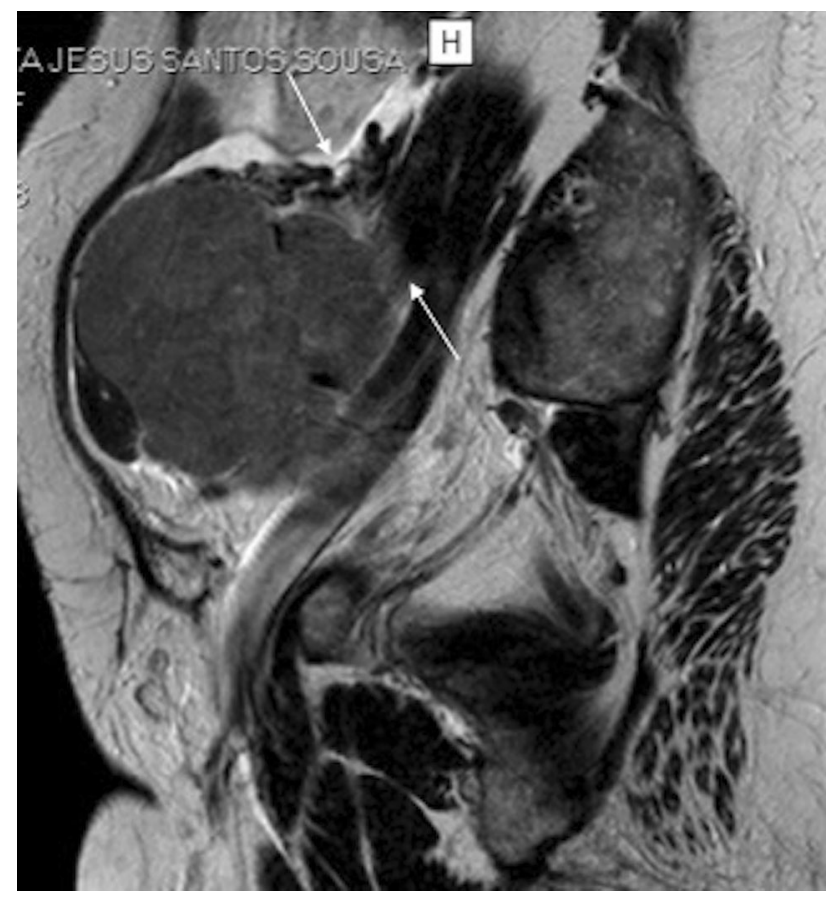

Fig. 13 "Ovarian suspensory ligament" sign caused by a Sertoli cell tumour of the right ovary. Sagittal T2-weighted image shows a fanshaped soft tissue structure anterior to the psoas muscles and in contiguity with the ovary (arrows). This ligament is crossed by the ovarian vessels to reach the ovarian hilum

The "embedded organ" sign means that at least a part of an organ seems to be embedded by the tumour [19]. On the contrary, organs that are adjacent to the mass and are not the site of origin, may display a crescent shape due to compression-“"negative embedded organ" sign (Fig. 10).
A large mass may also make a small organ such as the ovary, undetectable. This has been described as the "phantom organ" sign $[19,20]$.

The "beak" sign occurs when a mass deforms the edge of its organ of origin into a beak shape [19, 20] (Fig. 11).

"Prominent feeding artery" sign is seen when an artery is enlarged because of tumour vascular supply [19, 20]. Anatomical recognition of the dilated artery may allow the radiologist to diagnose the origin of the tumour (Fig. 12).

All of the described signs may be applied to both intraor extra-peritoneal masses [19, 20].

One sign that is used to determine the ovarian origin of a pelvic mass is the "ovarian vein" sign. The ovarian vein goes from the ovary through the ovarian suspensory ligament to the inferior vena cava (right ovarian vein) or to the left renal vein (left ovarian vein).

An enlarged ovarian vein is usually seen when a tumour arises from the ovary [21, 22]. The depiction of a dilated ovarian vein is also very useful to establish the laterality of the tumour, especially when the mass is very large and compresses the contralateral ovary. However, sometimes a large pelvic mass of non-ovarian origin can compress the ovarian vein and make it look like it is joining it [21,22]. For this reason, the "ovarian suspensory ligament" sign seems to be more reliable than the "ovarian vein" sign (Fig. 13).

The suspensory ligament of the ovary is a good anatomic landmark to find the ovary. It is a fold of peritoneum that connects the ovary to the pelvic wall. Both the ovarian artery and the ovarian vein cross the suspensory ligament of the ovary to reach the ovarian hilum. On axial images, it appears as a fan-shaped soft tissue structure anterior to the
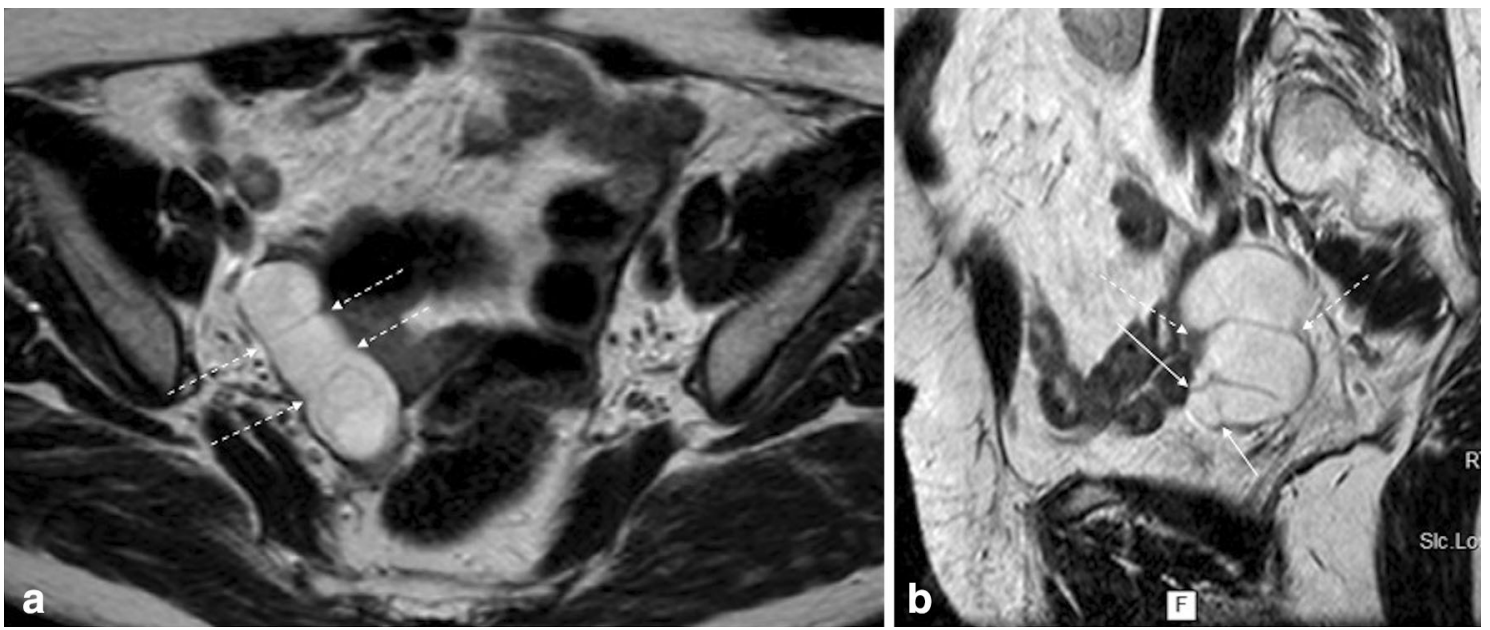

Fig. 14 "Waist" sign caused by a chronic hydrosalpinx in a 74-yearold woman. a Axial T2-weighted image. b Sagittal T2-weighted image. A well-defined tubular-shaped organ dilated with fluid is seen on both images. The depiction of incomplete folds (arrows) and of diametrically opposed indentations (dashed-arrows; "waist" sign) make the diagnose of a dilated hydrosalpinx very probable 

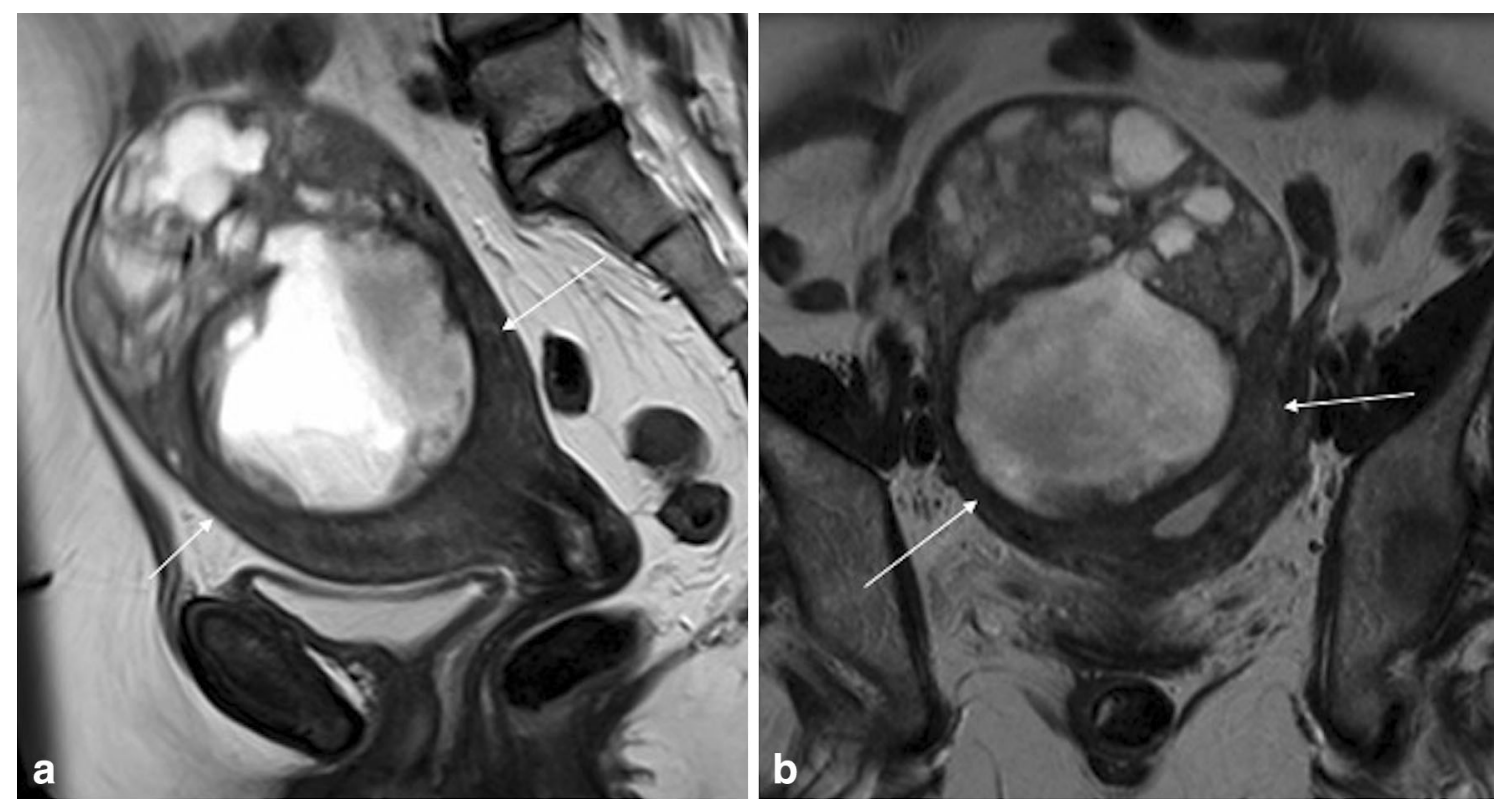

Fig. 15 "Claw" sign caused by a leiomyosarcoma in a 72-year-old woman. a Sagittal T2-weighted image. b Axial T2-weighted image. The "claw" sign describes the sharpened angles in the edge of a mass which correspond to the myometrium that surrounds a tumour of uterine origin

psoas muscle in continuity with the ovarian vessels that merges with the ovarian mass [22] (Fig. 13).

When a fallopian tube is dilated it appears as a welldefined, tubular-shaped organ with folded configuration into a $\mathrm{C}$ or a $\mathrm{S}$ (Fig. 14).

There are signs that may allow the radiologist to differentiate a dilated tube of an ovarian or para-ovarian cyst.

The visualization of incomplete longitudinal mucosal folds or plicae is a highly specific sign of a hydrosalpinx [23] (Fig. 14). The "waist" sign, which describes diametrically opposed indentations in the tube wall, can also help to confidently diagnose a dilated tube [24] (Fig. 14).

The "beads-on-a-string" and "cogwheel" signs were initially described in the set of salpingitis. However, they may also be found in tumoral tube disease (particularly the "cogwheel" sign). The "cogwheel" sign describes a dilated tube folded on itself, which results in the visualization of thick incomplete septa. The "beads-on-a-string" sign describes endosalpingeal folds, which when viewed on cross-section in a dilated tube simulate small nodular mural projections.

Although an uterine mass is usually promptly diagnosed, pedunculated leiomyomas sometimes are difficult to distinguish from broad ligament and adnexal masses (usually fibromas and thecomas). The misinterpretation especially occurs when large exophytic uterine degenerated leiomyomas or leiomyosarcomas make the visualization of the adnexa difficult (Fig. 15). In these cases, the differentiation from ovarian or tubal cancer might be difficult.
There are two radiological signs that may avoid this pitfall. The "bridging vessel" sign is present when we can clearly visualize the vessels anchoring the leiomyoma to the uterine wall (Fig. 13). The "claw" sign describes the sharpened angles in the edge of a mass, which correspond to the myometrium that surrounds the tumour of uterine origin (Fig. 15).

\section{Benign Gynaecological Entities Mimicking Ovarian Malignancy in a Non-emergency Setting}

\section{Fibroma and Cellular Fibroma}

Fibromas are benign lesions that account for approximately $4 \%$ of all ovarian tumours [25*0]. They tend to present in women in their late forties as hormonally inert tumours (Fig. 6).

The diagnosis of typical fibromas is usually straightforward. They range from small to large solid masses and characteristically present homogeneous low $\mathrm{T} 2$ signal and DWI signal $\left(b=800-1000 \mathrm{~s} / \mathrm{mm}^{2}\right)$. According to the latest ESUR guidelines, these features are enough to diagnose a lesion as benign [13••]. Fibromas usually also display weak and delayed contrast uptake [26•] (Fig. 6). Occasionally, they can present with speckled calcifications.

In our view, there are three situations that can present as potential pitfalls when accessing a fibroma.

Fibromas may be associated with exuberant ascites and hydrothorax (Meigs' syndrome) (Fig. 16). This 

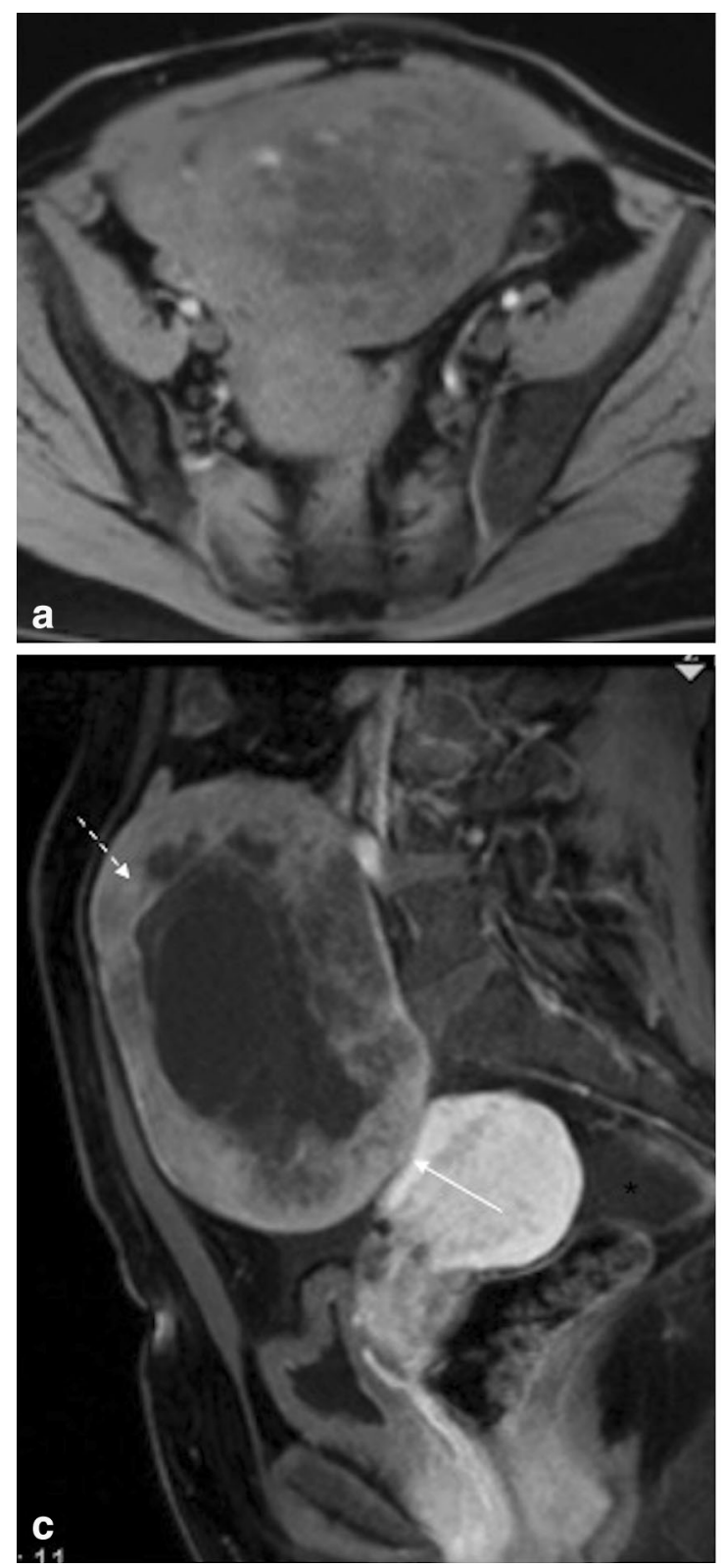

Fig. 16 Cellular fibroma of the right ovary in a 50-year-old female patient. a Axial fat-suppressed T1-weighted image; b Axial T2weighted image; c Sagittal fat-suppressed contrast-enhanced T1weighted image; d Sagittal T2-weighted image. A large, heterogeneous and well-defined tumour is seen making the "negative embedded organ" sign in the uterus (arrows). This supports its non-

combination of findings may lead the radiologist to diagnose a fibroma as a malignant tumour, since ovarian carcinoma typically has peritoneal spread.

Fibromas can sometimes have necrosis and haemorrhagic degeneration (Fig. 16). This is particularly true in cellular fibromas and when fibromas cause adnexal torsion.

Cellular fibromas are tumours with low malignant potential, representing approximately $10 \%$ of ovarian
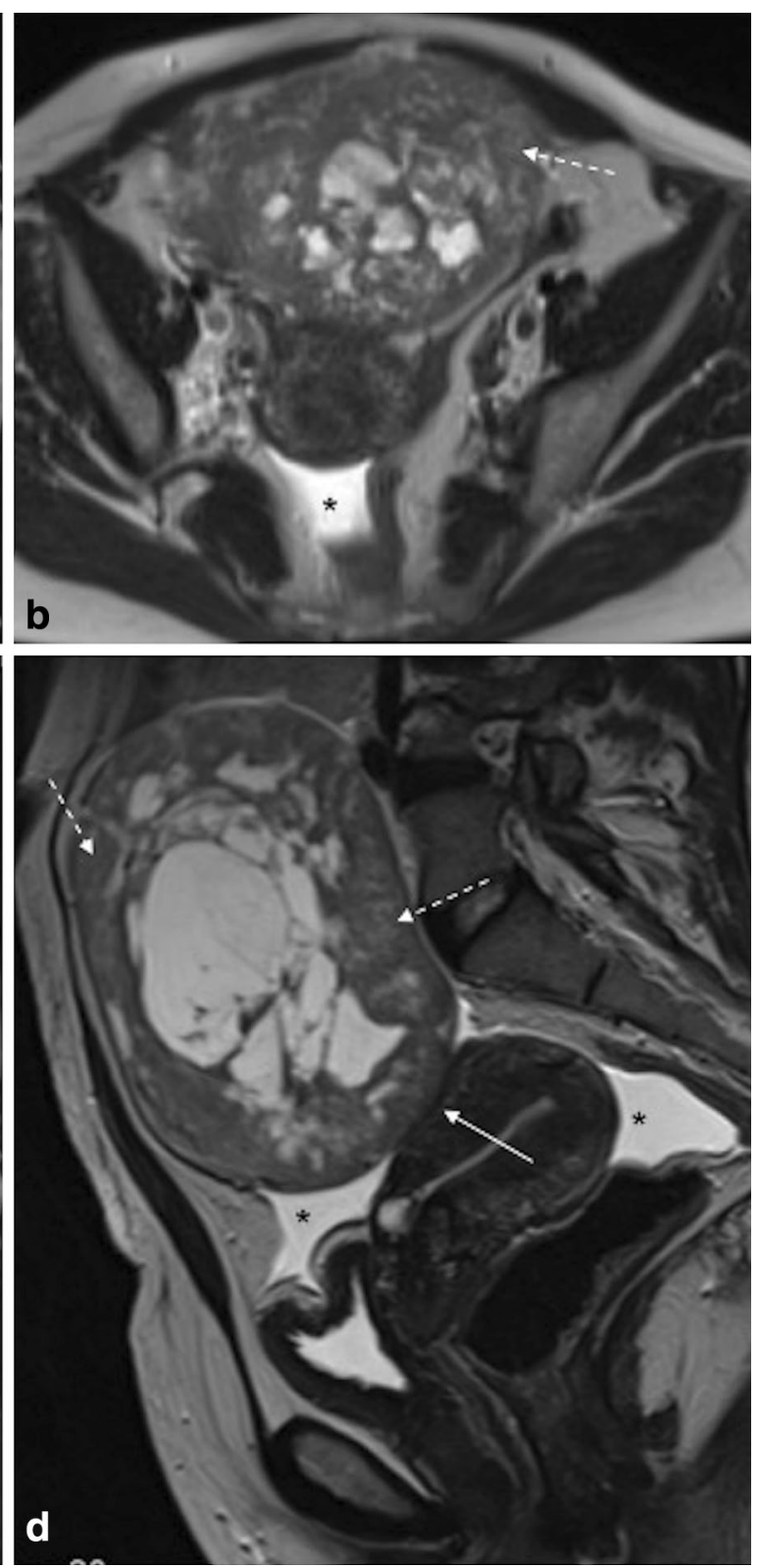

uterine origin. The tumour presents exuberant hyperintense T2weighted areas due to cystic degeneration. However, the solid areas of the tumour are intermediate to hypointense on T2-weighted images and show only mild contrast uptake when compared to the myometrium which are typical of fibromas (dashed-arrows). Ascites is also noted (asterisks)

fibromas [25••]. They usually present as large masses with heterogeneous signal due to haemorrhage and necrosis. A large mixed heterogeneous ovarian tumour with areas of T2 high signal or/and haemorrhagic content can be easily misdiagnosed as ovarian cancer (Fig. 16).

Any large ovarian mass can cause adnexal torsion. In a subacute setting, patients may present with non-specific symptoms and with an indeterminate solid adnexal mass on ultrasound. 

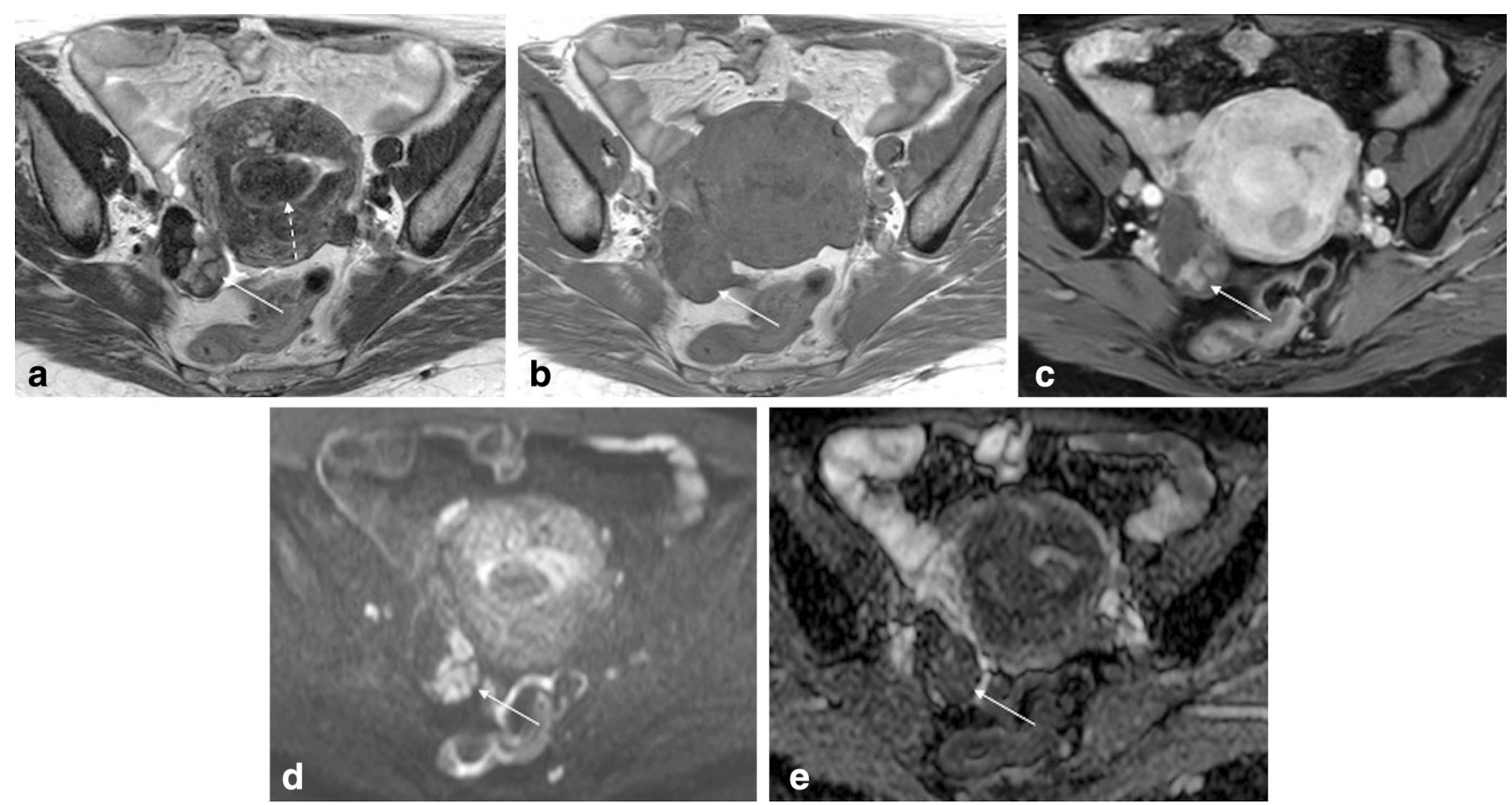

Fig. 17 Thecoma of the right ovary in a 75-year-old female patient. a Axial T2-weighted image; b Axial T1-weighted image; c Axial fatsuppressed contrast-enhanced T1-weighted image; $\mathbf{d}$ Axial diffusionweighted image $\left(b=1000 \mathrm{~s} / \mathrm{mm}^{2}\right) ;$ e ADC map. A small solid tumour is seen in the right ovary, showing both intermediate and hypo signal on T2-weighted images and moderate contrast uptake. The lesion also displays high signal on high $b$ values and low signal on ADC map. These characteristics may also be seen in ovarian cancer. No oestrogen-related symptoms were present in this case. A submucosal fundal leiomyoma is noted (dashed-arrow)
In both these scenarios, the radiologist should look for the presence of solid areas that display both low T2 signal and DWI signal $\left(b=800-1000 \mathrm{~s} / \mathrm{mm}^{2}\right)$ in order to make the correct diagnosis. If adnexal torsion is suspected, contrast administration is crucial to determine the non-viability of the ovary.

Fibromas are also frequently misdiagnosed as uterine leiomyomas. The discussion of this differential diagnosis will be done ahead (Fig. 16).

\section{Thecoma}

Thecomas are solid benign ovarian tumours that typically occur in post-menopausal women (Fig. 17). They are frequently associated with oestrogen-related symptoms. Therefore, women can present with uterine bleeding, endometrial hyperplasia and endometrial carcinoma.

The distinction between a thecoma and other malignant ovarian masses that may cause endometrial hyperplasia and/or cancer (endometrioid ovarian carcinoma; clear cell ovarian carcinoma; granulosa cell tumours) is usually easy; the latter are typically mixed ovarian masses. However, the differentiation from FIGO stage IIIA endometrial cancer may be challenging (invasion of the serosa and/or the adnexa through direct extension or metastases).
Endometrial cancer metastases to the ovaries may present as solid masses.

Pure thecomas do not have any particular characteristics and may mimic any malignant solid ovarian tumour.

Pure thecomas tend to display greater hyperintensity on T2-weighted images (due to oedema or cystic degeneration) and more avid contrast uptake when compared to more fibrous tumours, such as fibromas [27] (Fig. 17).

A study by Zhang et al. showed that most thecomas displayed homogenous isointense signal on T1- and T2weighted images and on DWI [28]. Moreover, the ADC values did not differ significantly from those of ovarian cancer [28] (Fig. 17).

\section{Cystadenofibroma and Brenner Tumour}

Both cystadenofibroma and Brenner tumour are epithelial ovarian tumours with fibrous components [25••] (Figs. 5, 18).

Cystadenofibromas are benign tumours that usually appear as complex cystic masses (Fig. 15). They sometimes show mild enhancing thick septa and/or nodular parietal thickening that may mimic the solid components of ovarian cancer. However, these solid components show very low signal on T2- weighted images as they are fibrous in nature (Fig. 5). 

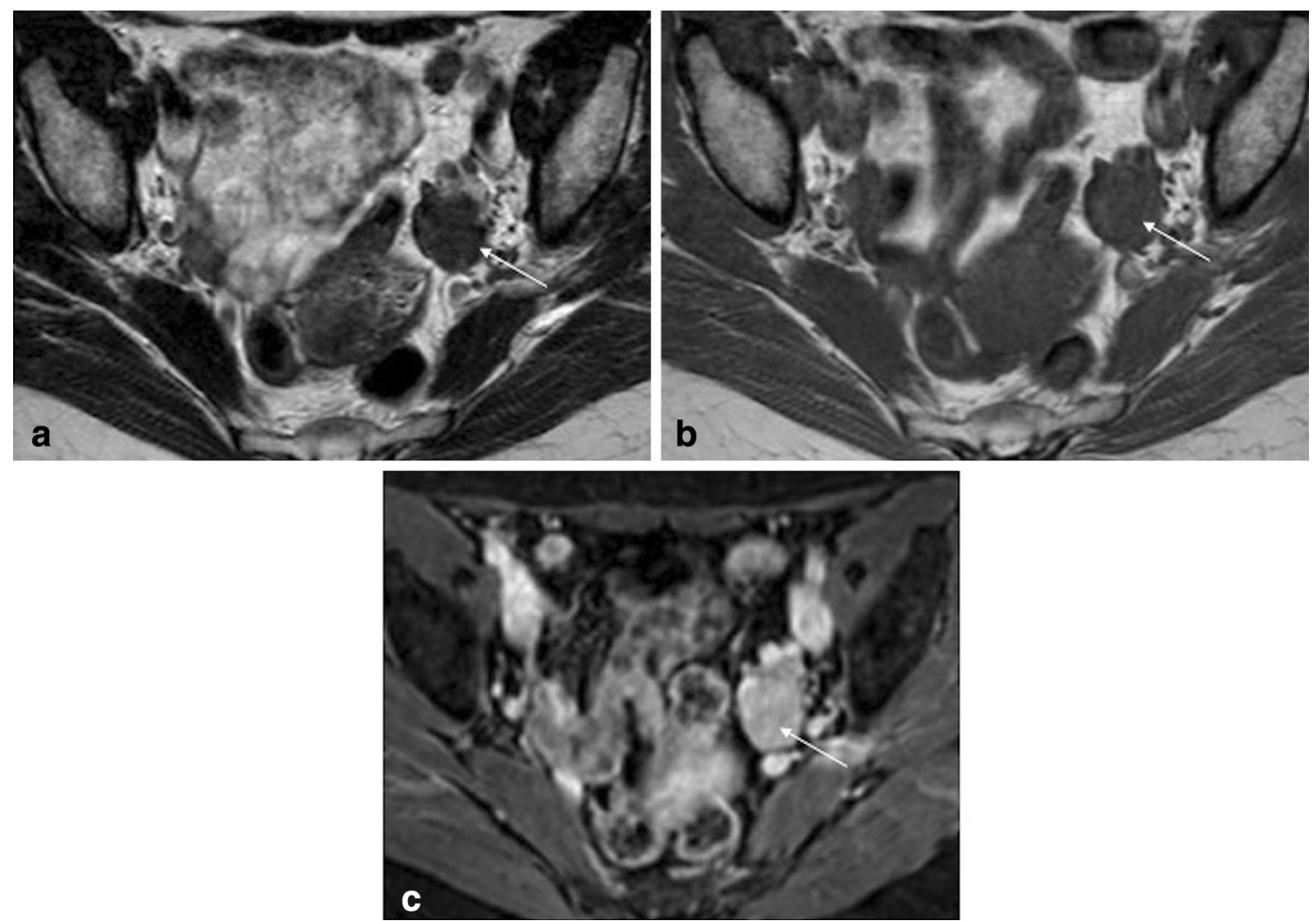

Fig. 18 Brenner tumour of the left ovary in a 58-year-old female patient. a Axial T2-weighted image; b Axial T1-weighted image; c Axial fat-suppressed contrast-enhanced T1-weighted image. A small solid tumour is seen in the left ovary, showing low signal on T2-weighted images but moderate contrast uptake

peripheral T2 hypo-/isointense areas with a spoke-wheel pattern and T2-hyperintense clefts (Fig. 2). Moreover, dynamic contrast-enhanced study typically reveals an avid and early peripheral uptake that progresses to central areas on delayed images. Sometimes a peripheral capsule may be seen, reflecting their slow-growing nature $[26 \bullet, 30]$ (Fig. 2).

\section{Struma Ovarii}

Struma ovarii is a monodermal teratoma that is predominantly or totally composed of thyroid tissue [25**] (Fig. 19). It is benign in the majority of cases; however thyroid malignancy arising in a struma ovarii has been reported [31].

On MR imaging, struma ovarii usually presents as a multiloculated mass, with locules of variable intensity and with solid components that may avidly enhance, mimicking malignancy [31] (Fig. 19). Diagnostic clues of this entity are: its typical "stained glass" appearance (due the variable content of its locules) and the presence of colloid material that characteristically shows hypointensity on both $\mathrm{T} 1$ - and T2-weighted images (Fig. 19). 

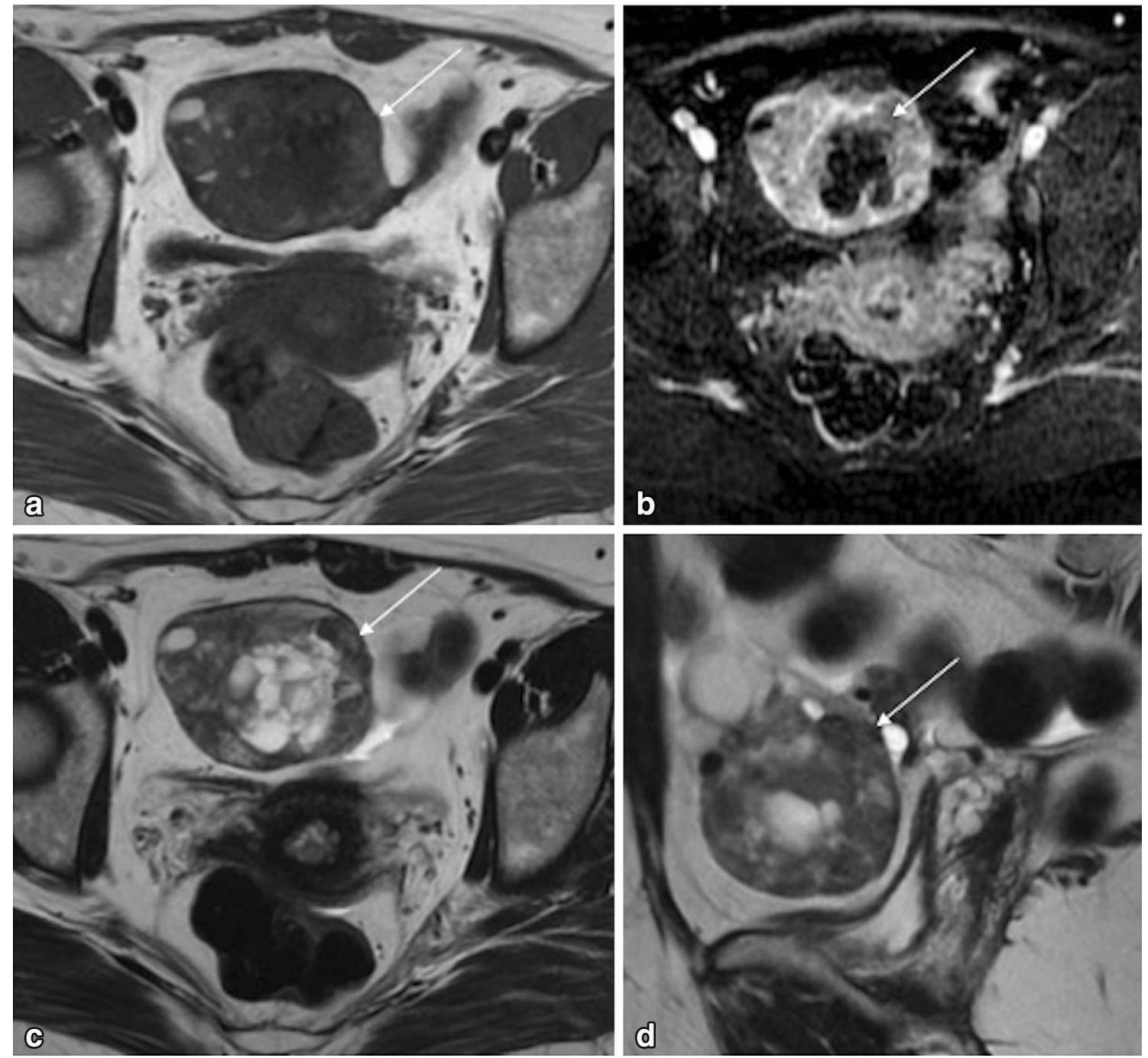

Fig. 19 Struma ovarii of the right ovary in a 46-year-old female patient. a Axial T1-weighted image; b Axial fat-suppressed contrastenhanced T1-weighted image; c Axial T2-weighted image; d Sagittal T2-weighted image. A multiloculated mass with locules of variable

\section{Degenerated Leiomyomas}

Typical leiomyomas usually do not pose diagnostic problems. They present as T2- hypointense uterine tumours with delayed contrast uptake. However, pedunculated subserosal degenerated leiomyomas may be difficult to differentiate from adnexal tumours (Fig. 3). This is particularly true when large masses impede the visualization of the ovaries and of the vascular pedicle between the uterus and the juxta-uterine mass. Furthermore, leiomyomas may detach from their stalk and form parasitic leiomyomas [32] (Fig. 20).

When a juxta-uterine mass is of unknown origin, a T2weighted oblique sequence should be performed along the long axis of the uterus to determine if it arises from the intensity and with solid components that avidly enhance is seen in the right ovary. Some of the locules show hypointense signal on both T1and $\mathrm{T} 2$-weighted images

ovary or from the uterus [13••, 32] (Fig. 20). Alternatively, a plane through the point of maximum contact of the mass and the uterus can also be done in order to look for the "bridging vessel" sign [13••].

About $10 \%$ of leiomyomas have histological degeneration [25••].This especially occurs in large tumours. Atypical degenerating and cellular forms of pedunculated leiomyomas with high signal on T2-weighted sequences can be very difficult to differentiate from ovarian cancer, especially when serum levels of CA-125 are elevated [32] (Fig. 20).

The types of degeneration that most resemble ovarian cancer are cystic, hyaline, myxoid and hydropic (Fig. 20). Although myxoid degenerating leiomyomas typically display high signal on T2-weighted sequences, they show 

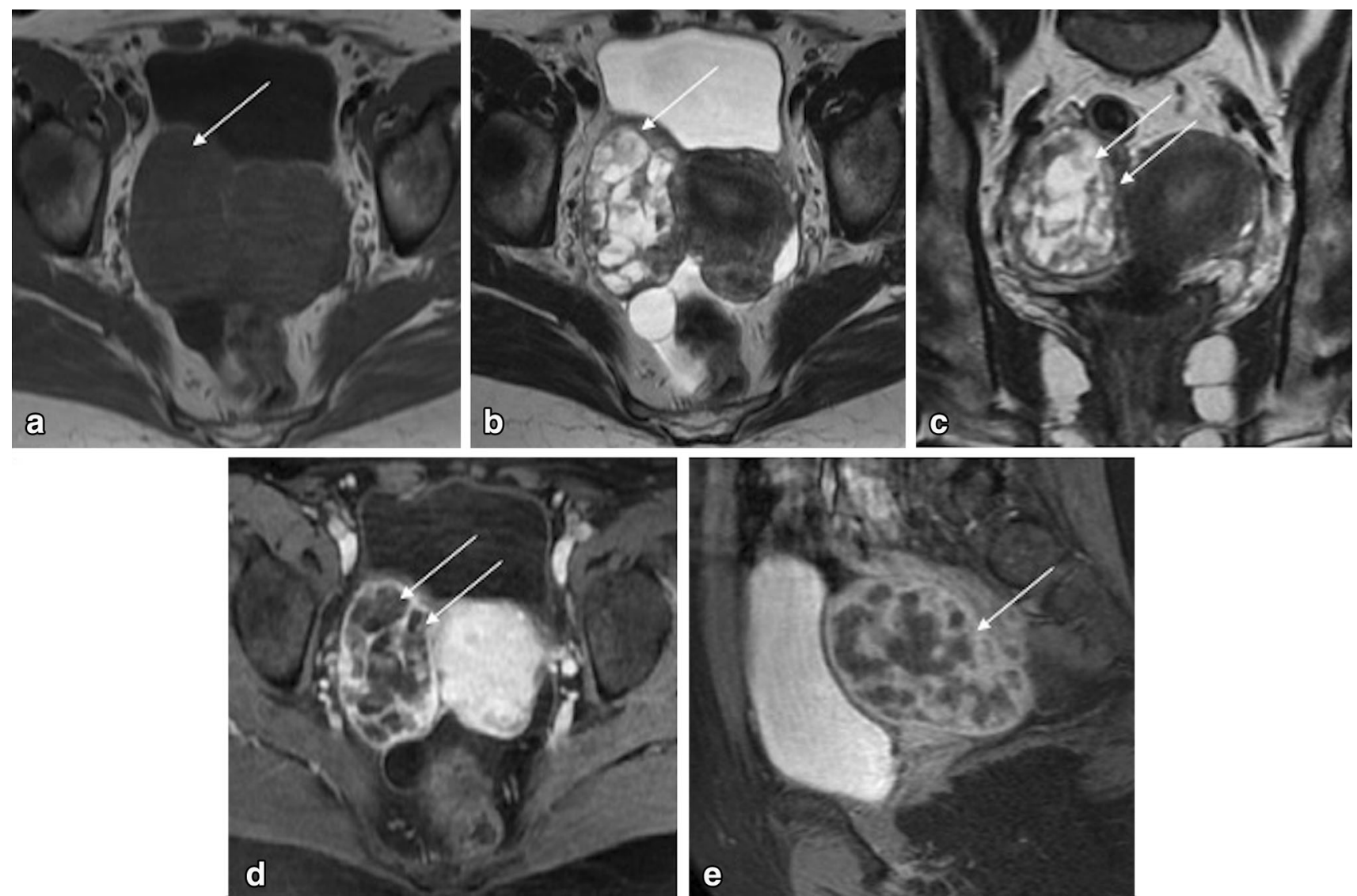

Fig. 20 Parasitic leiomyoma with cystic degeneration in a 47-yearold female patient. a Axial T1-weighted image; b Axial T2-weighted image; c Oblique T2-weighted image along the long axis of the uterus; $\mathbf{d}$ Axial fat-suppressed contrast-enhanced T1-weighted image; e Sagittal fat-suppressed contrast-enhanced T1-weighted image. A

mild contrast uptake that is not a feature of ovarian cancer. Hyaline leiomyomas usually show a cobblestone appearance, with both high and hyposignal areas on T2-weighted images.

Diffuse hydropic leiomyomas are rare. Since they have abundant watery oedema, high T2-signal can be misinterpreted as myxoid matrix or as malignancy (of uterine or ovarian origin) [32]. Moreover, they may also be associated with "Pseudo-Meigs" syndrome, another potential confounder.

\section{Conclusion}

MRI provides high resolution images and it is a powerful imaging modality for the diagnosis of female pelvic masses of indeterminate origin.

It can be very challenging to define the correct origin of a pelvic mass. The radiologist should try to look for anatomic signs that may help to diagnose the mass as intra- or tumour with cystic degeneration is seen next to the uterus. The oblique T2-weighted image along the long axis of the uterus clearly shows that the tumour has another origin. If the ovaries are not clearly depicted, a parasitic leiomyoma with cystic, hyaline or myxoid degeneration can be easily misinterpreted as an ovarian cancer extra- peritoneal. Then, the radiologist should try to find specific anatomical organ-signs to define the mass as ovarian or non-ovarian in origin.

When ovarian origin is suspected, the radiologist should be aware of benign entities that mimic malignancy. In a nonemergency setting, they include: fibromas, cellular fibromas, thecomas, cystadenofibromas, Brenner tumours, sclerosing stromal tumours, struma ovarii and degenerated leiomyomas.

Knowledge of the existence of these diagnostic pitfalls should help the radiologist to avoid misinterpretation of lesions in a pelvic magnetic resonance performed for the characterization of an indeterminate female pelvic mass.

\section{Compliance with Ethical Guidelines}

Conflict of interest Mariana Horta and Teresa Margarida Cunha each declare no potential conflicts of interest.

Human and Animal Rights and Informed Consent This article does not contain any studies with human or animal subjects performed by any of the authors. 


\section{References}

Papers of particular interest, published recently, have been highlighted as:

- Of importance and

-• Of major importance

1. $\bullet$ Bruno MA, Walker EA, Abujudeh HH. Understanding and confronting our mistakes: the epidemiology of error in radiology and strategies for error reduction. RadioGraphics. 2015;35(6): 1668-76. Recent review article about different types of errors in Radiology and about strategies to reduce them.

2. Kravitz RL, Rolph JE, McGuigan K. Malpractice claims data as a quality improvement tool. I. Epidemiology of error in four specialties. JAMA. 1991;266(15):2087-92.

3. Abujudeh HH, Boland GW, Kaewlai R, Rabiner P, Halpern EF, Gazelle GS, et al. Abdominal and pelvic computed tomography (CT) interpretation: discrepancy rates among experienced radiologists. Eur Radiol. 2010;20(8):1952-7.

4. Lee CS, Nagy PG, Weaver SJ, Newman-Toker DE. Cognitive and system factors contributing to diagnostic errors in radiology. AJR Am J Roentgenol. 2013;201(3):611-7.

5. Berlin L. Radiologic errors and malpractice: a blurry distinction. AJR Am J Roentgenol. 2007;189(3):517-22.

6. Muhm JR, Miller WE, Fontana RS, Sanderson DR, Uhlenhopp MA. Lung cancer detected during a screening program using four-month chest radiographs. Radiology. 1983;148(3):609-15.

7. Harvey JA, Fajardo LL, Innis CA. Mammograms with impalpable retrospective in breast vs blinded previous patients carcinoma: 1993 ARRS President's award. AJR Am J Roentgenol. 1993;161(6):1167-72.

8. Berlin L, Hendix RW. Perceptual errors and negligence. AJR Am J Roentgenol. 1998; 170:863-7.

9. Asch E, Levine D, Pedrosa I, Hecht JL, Kruskal J. Patterns of misinterpretation of adnexal masses on CT and MR in an academic radiology department. Acad Radiol. 2009;16(8):969-80.

10. • Woo YL, Kyrgiou M, Bryant A, Everett T, Dickinson HO. Centralisation of services for gynaecological cancer. Cochrane Database Syst Rev. 2012; 14;(3):CD007945. Meta-analysis to assess the effectiveness of centralisation of care for patients with gynaecological cancer.

11. • Lakhman Y, D’Anastasi M, Miccò M, Scelzo C, Vargas HA, Nougaret $\mathrm{S}$, et al. Second-opinion interpretations of gynecologic oncologic MRI examinations by sub-specialized radiologists influence patient care. Eur Radiol. 2016; 26(7):2089-98. Recent study that determined the impact of second-reading of gynaecological MRI by sub- specialized gynaecological radiologists.

12. Harris RD, Javitt MC, Glanc P, Brown DL, Dubinsky T, Harisinghani MG, et al. ACR appropriateness criteria? Clinically suspected adnexal mass. Ultrasound Q. 2013;29(1):79-86.

13. • Forstner R, Thomassin-Naggara I, Cunha TM, Kinkel K, Masselli G, Kubik-Huch R, et al. ESUR recommendations for MR imaging of the sonographically indeterminate adnexal mass: an update. Eur Radiol. 2017; 27(6):2248-57. European Society of Genitourinary Radiology updated guidelines for sonographically indeterminate adnexal masses.

14. Thomassin-Naggara I, Daraï E, Cuenod CA, Fournier L, Toussaint I, Marsault C, et al. Contribution of diffusion-weighted MR imaging for predicting benignity of complex adnexal masses. Eur Radiol. 2009;19(6):1544-52.
15. Thomassin-Naggara I, Dara E, Cuenod CA, Rouzier R, Callard P, Bazot M. Dynamic contrast-enhanced magnetic resonance imaging: a useful tool for characterizing ovarian epithelial tumors. J Magn Reson Imaging. 2008;28(1):111-20.

16. Bernardin L, Dilks P, Liyanage S, Miquel ME, Sahdev A, Rockall A. Effectiveness of semi-quantitative multiphase dynamic contrast-enhanced MRI as a predictor of malignancy in complex adnexal masses: radiological and pathological correlation. Eur Radiol. 2011;22(4):880-90.

17. Thomassin-Naggara I, Aubert E, Rockall A, Jalaguier-Caudray A, Rouzier R, Darai E, et al. Adnexal masses: development and preliminary validation of an MR imaging scoring system. Radiology. 2013;267(2):432-43.

18. Horta M, Couceiro C. Peritoneal anatomy: imaging overview. ESGAR 2013.e-Poster:EE-113.

19. Dionisio T, Gomes FV, Cunha TM. Non-ovarian pelvic masses: key concepts and useful clues to the radiologist. Eur Congr Radiol. 2014. doi:10.1594/ecr2014/C-1191.

20. Nishino M, Hayakawa K, Minami M, Yamamoto A, Ueda H, Takasu K. Primary retroperitoneal neoplasms: CT and MR imaging findings with anatomic and pathologic diagnostic clues. RadioGraphics. 2003;23(1):45-57.

21. Lee JH, Jeong YK, Park JK, Hwang JC. "Ovarian vascular pedicle" sign revealing organ of origin of a pelvic mass lesion on helical CT. AJR Am J Roentgenol. 2003;182(1):131-7.

22. Saksouk FA, Johnson SC. Recognition of the ovaries and ovarian origin of pelvic masses with CT. RadioGraphics. 2004;24(suppl_1): S133-46.

23. Outwater EK, Siegelman ES, Chiowanich P, Kilger AM, Dunton CJ, Talerman A. Dilated fallopian tubes: MR imaging characteristics. Radiology. 1998;208(2):463-9.

24. Patel MD, Acord DL, Young SW. Likelihood ratio of sonographic findings in discriminating hydrosalpinx from other adnexal masses. AJR Am J Roentgenol. 2006;186(4):1033-8.

25. • Kurman RJK, Carcangiu ML, Herrington CS, Young RH. Classification of tumours of the ovary. In: WHO Classification of Tumours, Volume 6. 4th ed. Lyon: IARC, 2014; 11-83. Latest WHO histological classification of gynaecological tumours.

26. - Horta M, Cunha TM. Sex cord-stromal tumors of the ovary: a comprehensive review and update for radiologists. Diagn Interv Radiol. 2015;21(4):277-86. Most recent radiological review of sex cord-stromal tumours of the ovary.

27. Tanaka YO, Tsunoda H, Kitagawa Y, Ueno T, Yoshikawa H, Saida Y. Functioning ovarian tumors: direct and indirect findings at MR imaging. RadioGraphics. 2004;24(suppl_1):S147-66.

28. Zhang H, Zhang G-F, Wang T-P, Zhang H. Value of $3.0 \mathrm{~T}$ diffusion-weighted imaging in discriminating thecoma and fibrothecoma from other adnexal solid masses. J Ovarian Res. 2013;6(1):1.

29. Outwater EK, Wagner BJ, Mannion C, McLarney JK, Kim B. Sex cord-stromal and steroid cell tumors of the ovary. Radiographics. 1998;18(6): 1523-46.

30. Jung SE, Rha SE, Lee JM, et al. CT and MRI findings of sex cord-stromal tumor of the ovary. Am J Roentgenol. 2005;185(1): 207-15.

31. Leite IST, Cunha TM, Figueiredo JP, Félix A. Papillary carcinoma arising in struma ovarii versus ovarian metastasis from primary thyroid carcinoma: a case report and review of the literature. J Radiol Case Rep. 2013;7(10):1-10.

32. Horta M, Cunha TM, Oliveira R, Magro P. Hydropic leiomyoma of the uterus presenting as a giant abdominal mass. BMJ Case Rep. 2015;8:bcr2015211929-5. 